\title{
PCCP
}

ARTICLE TYPE

Cite this: DOI: $00.0000 / x x x x x x x x x x$

\section{Towards peptide-based tunable multistate memristive materials ${ }^{\dagger}$}

\author{
Salvador Cardona-Serra*a, Lorena E. Rosaleny ${ }^{* a}$, Silvia Giménez-Santamarina ${ }^{a}$, Luis \\ Martínez-Gil $^{b}$, and Alejandro Gaita-Ariño*a
}

Received Date

Accepted Date

DOI: $00.0000 / x x x x x x x x x x$

\begin{abstract}
Development of new memristive hardware is a technological requirement towards widespread neuromorphic computing. Molecular spintronics seems a fertile field for the design and preparation of this hardware. Within molecular spintronics, recents results on metallopeptides demonstrating the interaction between paramagnetic ions and the Chirality Induced Spin Selectivity effect hold particular promise for developing fast (ns- $\mu \mathrm{s}$ ) operation times. [Torres-Cavanillas et al, J.Am.Chem.Soc., 2020, DOI:10.1021/jacs.0c07531]. Among the challenges in the field, a major highlight is the difficulty in modelling the spin dynamics in these complex systems, but at the same time the use of inexpensive methods has already allowed progress in that direction. Finally, we discuss the unique potential for biomolecules for the design of multistate memristors with a controlled -and indeed, programmablenanostructure, allowing to go beyond anything that is conceivable employing conventional coordination chemistry.
\end{abstract}

\section{The need for molecular memristors}

Complementary Metal-Oxide-Semiconductor (CMOS) technology and the von Neumann architecture, $\frac{1}{1}$ constitute the fundamental structure of modern computers and are the basis of virtually all computing systems. Within the approach proposed by von Neumann in 1945, a computer is composed of two basic and separated elements, the processing and the memory units. The physical separation between these two kinds of elements limits the efficiency of this architecture in the processing speed of large amounts of information. This effect, known as the von Neumann bottleneck, ${ }^{2}$ hinders applications such as pattern recognition and big data. Mitigation efforts are underway, including a search for more efficient computing models. However, humans already have -and employ- the most efficient computer in terms of speed and power consumption: the biological brain. With only $20 \mathrm{~W}$ of power consumption, $\frac{3}{3}$ the brain is able to process and classify massive environmental information much more efficiently than any artificial device up to date. This high-performance processing results from the inner workings of the neurons, as they can

\footnotetext{
a ICMol, Universitat de València, Paterna, Valencia, Spain.Tel: +34 963544155; Email: salvador.cardona@uv.es, lorena.rosaleny@uv.es, alejandro.gaita@uv.es

${ }^{b}$ Dept. de Bioquímica y Biología Molecular, Universitat de València, Paterna, Valencia, Spain.

$\dagger$ Electronic Supplementary Information (ESI) available:

S1 Distance and relative orientation of two lanthanide coordination centers in daLBT S2 Bacterial expression of LBT peptides

S3 Design of a nonanuclear metallopeptide

S4 Further approaches for the biochemical design of polynuclear metallopeptides. See DOI: $10.1039 / \mathrm{cXCP00000x/}$
}

combine information processing with memory storage in a single component. This has inspired a huge effort towards Neuromorphic Computing: mimicking the behavior of neurons by means of electronic components and building Artificial Neural Networks. In this context, memristors are ideal materials for neurohardware applications due to its ability to retain and process information like neurons do.4

The memristor is a fundamental and irreducible component in the theory of modern electronic circuits. On its original formulation in 1971, Chua theoretically formulated a hypothesis about the existence of the memristor as a fourth passive electronic component, at the level of the inductors, resistors and capacitors. This component arose from a generalization of Ohm's law:

$$
v(t)=M(q(t)) \cdot i(t)
$$

where the symbols indicate time evolution of: voltage $(v(t))$, current intensity $(i(t))$ and charge $(q(t))$ through the device. $M$ is the magnitude representing the memristivity.

In practical terms, the characteristic that defines a memristive material is its ability to vary non-linearly its resistance according to previous history of voltage applied to it over a period of time, resulting in the distinctive fingerprint of featuring a pinched hysteresis loop in I-V plots, which can display a variety of shapes and thus give rise to a variety of applications (see Fig. 11, up). This property called memristivity has been proposed to be the foundation of vanguard computer science, from the design of new Resistive RAM (RRAM), that can surpass current Flash Disks and HDDs, to the opening of new in-memory computing paradigms. 6 
Integrated memristive devices are considered promising candidates for these applications because they are able to both store and process information, being able to build neuromorphic units at the nanometer scale which ensures to reduce, at the same time, the energetic cost and the size. ${ }^{7}$

The first memristive material was proposed as late as in 2008 in HP laboratories, and was based on oxygen migration mechanism in the interface between $\mathrm{TiO}_{2} / \mathrm{TiO}_{2-x} \cdot{ }^{[8}$ In this specific case, the interface between both inorganic phases acts as a mobile barrier where oxo anions move as a function of the current passing through the device. The relative position of the barrier between $\mathrm{TiO}_{2} / \mathrm{TiO}_{2-x}$ results in different states of resistance. This mechanism gives rise to the so-called 'memristive switching' effect. A more recent type of memristive materials uses nanoscale spintronic oscillators, a totally different approach, where magnetism and electronics interplay for building the neuronal units. 9 This route is based on the concept of spin-valve magnetoresistance, where the total resistance of the Magnetic Tunnel Junction (MTJ) depends on the relative orientation of a soft-variable ferromagnet with respect to a hard-fixed ferromagnet. 10 Thus, the current passing through the junction generates a torque on the magnetization of the soft-ferromagnet which leads to a spin precession with frequencies varying from $100 \mathrm{MHz}$ to tens of GHz. These spin precessions can be converted to voltage oscillations through magnetoresistance. ${ }^{11}$ Due to their response to thermal noise, MTJ have been presented as non-volatile magnetic memories that can contribute with a certain stochasticity in the resistance transition. This property has been recently exploited for random number generation and other noise-based computing applications. $\frac{12}{12}$ While this spintronics-based mechanism is physically very different from the oxygen ion migration described above, substantially the result is the same: a process whereby electric current through a component results in a controllable change of electrical resistance. Indeed, a variety of physical processes can give rise to analogous memristive behaviors, and different materials have been found to be more or less adequate for different memristive applications (see Fig. 1). 1314

Despite the availability of plenty of extended inorganic materials displaying memristive behavior, practical feasibility is inherently limited by low chemical variability and possible functionalizations, difficulties in nanostructuration, poor reproducibility because of large parameter dependencies and limited cycling endurance.1819 Alternatively, molecular materials can display memristivity and potentially excel in some aspects when compared with traditional inorganic materials. First, they allow using the versatility of molecular chemistry to design new materials or enhance its physical properties. Second, it permits to reduce the size and the energetic requirements. ${ }^{20}$ Third, it improves the processability by means of low-cost soft techniques. 21 23 Here, we mention the three molecular memristive mechanisms proposed so far: (i) Ionic transport-based,, 17 (ii) Redox-based ${ }^{16}$ and (iii) Spintronic-based. 15 An evaluation of the main parameters that characterize the suitability of these mechanisms is depicted in a spider chart in Fig. 1 down. The strategy proposed in this work is encompassed in the green category of spintronic-based memristive materials and is strongly related to MTJ and the spin torque
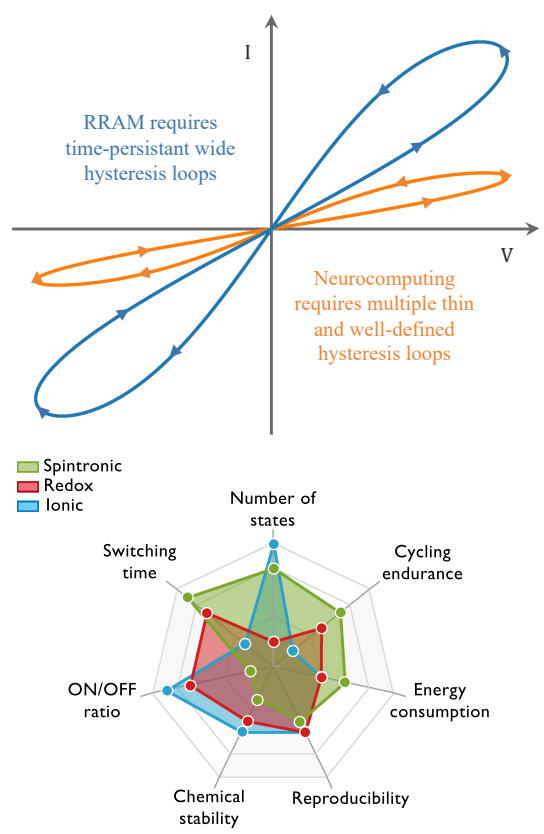

Fig. 1 (Up) Typical pinched hysteresis loops in terms of I-V cycles for: (orange curve) memristive materials with lower retentention times, thinner hysteresis loops and, probably, higher number of resistive states which will be more suitable for building a synaptic dynamic element; contrary to (blue curve) memristive materials with higher retention times and with lower number of resistive states, more suitable for long memory applications. (Down) Illustration of the expected critical features for three possible molecular memristive mechanisms. Green: Spintronic-based ${ }^{15}$ (the focus of the present work), red: Redox-based, $\underline{16}$ blue: lonic transportbased. 17

mechanism aforementioned.

\section{Metallopeptides: the spintronics pathway to- wards biomolecular memristors}

Biomolecules have already been explored for non-spintronicbased memristive applications, and there are even proposals for biomaterial-based artificial synapses, employing materials such as lignin, collagen and $t$-carrageenan, 24 although most of the current research is still focused on the use of extended inorganic materials. 24.27 Herein we will discuss the progress so far in the exploration of biomolecules for the design and preparation of molecular memristors exploiting the spintronic strategy. In particular, we are interested in a phenomenon called Chirality Induced Spin Selectivity (CISS) that is responsible of a notable spin polarization at room temperature and without the presence of magnets, when current passes through chiral molecules. ${ }^{28} 29$ In fact, in the last decade chirality-based spintronic research has succeeded in obtaining molecular spin valves that operate in absence of a hard magnet. In this case, the hard ferromagnetic layer is replaced by a chiral helix which originates the spin polarized current due to the spin filtering produced by the CISS effect. 30 .31 Within this approach, the magnetic moment of a single-domain ferromagnetic platelet or nanoparticle gets oriented by the spin polarized current using the spin torque effect. This achievement supposed a milestone which confirmed that electronic memory 
elements based on molecular CISS effect could be fabricated. A further extension of this idea to build neuromorphic units using molecular chiral materials has been proposed. Particularly, in the case of Bustami et. al, 15 the conductance of the material was manipulated by inverting the current passing through the platelet and thus modifying the spin current of the interface. In this experiment, the metallic contacts, coated with chiral molecules, exhibit a coercive field which makes them extremely sensitive to the spin current magnetization. This work suggested, for the first time, to build memristive memories by introducing CISS-effect in a cutting-edge discipline. Although this work showed that its use as a resisitive RAM (RRAM) is mostly feasible, its computing capabilities, however, are restricted due to a long magnetization permanence. As we will see below, using a single paramagnetic ion instead of a larger single-domain moiety would allow to reduce drastically the computing times, opening the door to certain synaptic applications. 32

The CISS effect has been already demonstrated in biomolecules, from modified aminoacids like L- or D-stearoyl lysine ${ }^{33}$ and simple peptides like polyalanine, 34 to proteins like bacteriorhodopsin ${ }^{[35 \mid 36}$ or double-stranded DNA. $\stackrel{37}{ }$ However this has almost exclusively been done for diamagnetic molecules. On the other hand, spintronics experiments show that it is also possible to obtain spin-dependent transport through nonchiral, nonbiological molecules with a single paramagnetic ion. 3839

By combining chiral paramagnetic polypeptides with the concept of spin-valve magnetoresistance, one can have a paramagnetic ion -or, as we will see below, more than one- playing the role of the soft magnet whereas the chirality of the molecule, via the CISS effect, plays the role of the hard magnet, with the combination of both achieving a memristance that depends on the previous voltage history of the component (see Fig. 2). Obtaining chiral molecules that are at the same time polynuclear metal complexes with specific coordination sites would be a daunting chemical challenge, but a unique feature of biomolecules is the possibility of self-organization into highly complex structures, including in this case polynuclear, magnetic, chiral peptides. This points toward the possibility of using biomolecules for the organization of multistate memristive building blocks (see Fig. 2). Choosing biomolecules in this context would open the door to multiscale complexity, permitting the use of techniques developed in molecular biology, including recombinant protein technology 40 or CRISPR-Cas9. 41 These techniques are commercially available and enable obtaining on-demand peptide sequences and modifications of existing proteins inexpensively, facilitating tailored molecular modification and multiscale organization as we will explore in the next section.

Employing paramagnetic biomolecules as memristive components is a general strategy that can conceivably be implemented in many different ways: any metalloprotein is in principle a candidate, as are spin-tagged peptides and proteins. 42.43 Equivalently, DNA origami 44 could be used for this same goal. Here we highlight the possibility of using the interaction between the magnetic polarized current (obtained by CISS effect) and the magnetic momentum of the coordinated lanthanoid to obtain high-frequency memristive behavior. The experimental strategy considers a flux

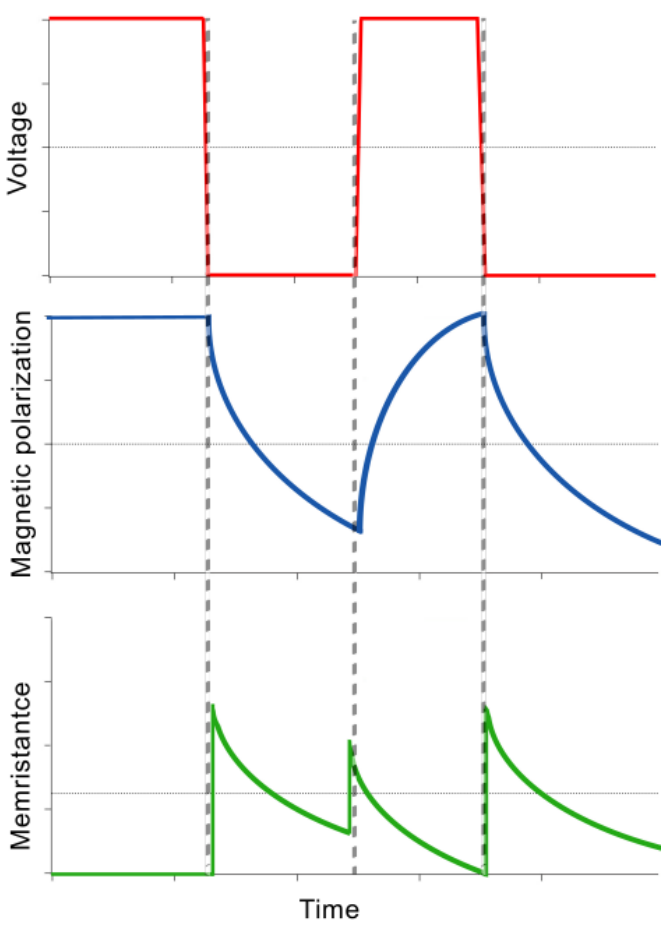

Fig. 2 Schematic functioning of a spintronics multistate memristive device based on the CISS effect in a paramagnetic molecular material. Let us start the experiment with the voltage in the ON position for an unspecified but long enough time to guarantee that the magnetic polarization of the metal ions is maximal due to the interaction with the CISS-polarized current, and the resistance is correspondingly minimal. A sudden switch in the voltage to the OFF position, e.g. a change in sign, has two consequences: it starts a relatively slow process of relaxation of the magnetic polarization, and it instantly increases the memristance to its maximum value. This sudden jump in the memristance is due to the "wrong" polarization of the paramagnetic ions, which now opposes the spin polarization of the CISS current. As the magnetic polarization evolves towards its new equilibrium situation, the memristance decreases too, accessing a continuum of values, i.e. behaving as a multistate memristance. Depending on the timing of the subsequent changes in the voltage between the ON and OFF values, the time evolution of the magnetic polarization of the system and of the memristance of the device will present different shapes; a fine time-control of the voltage allows to achieve any desired memristance between the ON and OFF limit values.

of spin-polarized current injected through an helical molecule, considering that the magnetic exchange will effectively polarize this ion with respect to the current. The key to understand how an avenue to novel nanospintronics is opened by these complexes requires thinking about the involved time scales (see Fig. 2). At long times, the average magnetic polarization of the lanthanide ion will be determined by the direction of the current, but as long as one is operating at high enough frequencies $(>1 \mathrm{GHz})$ it will be determined by the voltage history instead. Since the sign of the lanthanoid magnetic polarization affects the conductivity, this means that the conductance of the device effectively depends on the applied voltage history. In other words, the combination of a non-negligible magnetic exchange with the CISS effect would produce a strong magnetoresistance that includes short-time memory effects. In such a setup, memristor-like nonlinear logic operations at low voltages under ambient conditions and room temperature 
would be expected. This opens the door to obtain an all-organic single molecule memristor. As an illustration and because of our recent positive results with it, here we focus on a particular helical structure: lanthanide ions coordinated by short peptide sequences (15-17 aminoacids) known as Lanthanide Binding Tags (LBT) (see Fig. 3 left). $\stackrel{45}{4}$ These $\mathrm{Ln}^{3+}$-quelating ligands were originally optimized by Imperiali et al. via the exploration of a series of peptide libraries, starting from $\mathrm{Ca}^{2+}$-binding motifs of proteins, with the combined goals of fine-tuning the selectivity of binding to lanthanide ions and presenting photoluminescence. 46 As any short peptide, LBTs can easily be genetically encoded, thus they are used as luminescent spin tags in controlled protein positions, 47 and a double tagging has also been used to perform nondisruptive in-cell structural measurements via Double ElectronElectron Resonance (DEER). 48

Following this idea, we recently showcased the combination of these features in the first report of spin filtering in a solid-state device based on a self-assembled monolayer prepared with $\mathrm{Tb}^{3+}$ coordinated by an LBT peptide. ${ }^{49}$ The active role of the paramagnetic ion was unambiguously confirmed by three independent experimental approaches: cyclic voltammetry (see Fig. 3 right), electrochemical impedance spectroscopy and local transport in solid state devices using liquid-metal drop contacts (see Fig. 4). In the best conditions, a spin polarization $S P=\frac{J_{u p}-J_{\text {down }}}{J_{u p}+J_{d o w n}} \times 100=$ $-70 \pm 10$ was achieved with the contribution of complexed $\mathrm{Tb}^{3+}$ ions, meaning the current density $J$ changed significantly depending on the spin polarization of the current (see Fig. 4). This value is close to the maximum spin filtering reported up to date with the extra advantage of manipulating both filtering sources independently. $\cdot \frac{50151}{1}$ The next step would be to perform time-dependent measurements at high enough frequencies to be affected by spin dynamics.

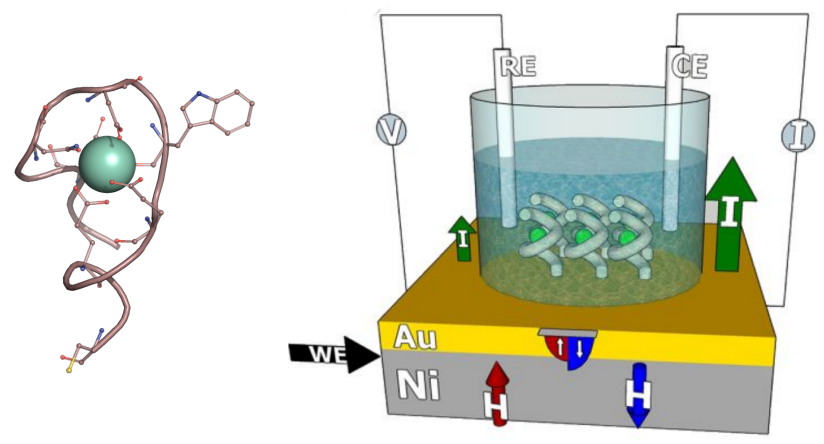

Fig. 3 Left: Structure of the LBT peptide complexed with $\mathrm{Tb}^{3+}$ (green sphere). Right: Depiction of one of the the spintronic devices used to detect the interaction between CISS and the paramagnetic $\mathrm{Tb}^{3+}$ ion (reproduced with permission from 49 ).

The potential advantages of the approach we propose are directly related with the design possibilities of magnetic peptides: (a) switching time, over which we have some control via the spin dynamics that in lanthanides are governed by the coordination sphere and (b) number of states, where a higher number of achievable states correspond to a better synaptic element. This
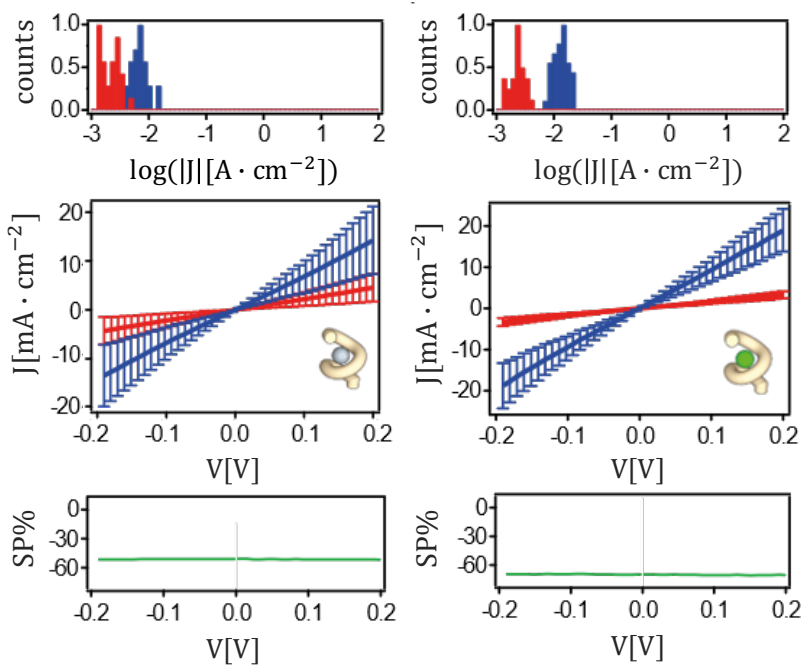

Fig. 4 Time-independent spin filtering due to Chirality Induced Spin Selectivity, showing how the effect is enhanced in presence of a coordinated paramagnetic ion (adapted with permission from ${ }^{49}$ ). Left: LBT coordinating diamagnetic $\mathrm{Y}^{3+}$ Right: LBT coordinating paramagnetic $\mathrm{Tb}^{3+}$. Top: current density histograms. Center: average current density vs voltage curves measured under an external magnetic field. Bottom: Spin polarization as a function of the bias voltage. Red and blue curves correspond to an external magnet pointing up or down respectively.

can be also completed with a low power consumption, which is always present while working with spin-electronic degree of freedom versus the usual electronic-only information. Finally an apparent disadvantage, in comparison with the redox and ion transport mechanisms (see Fig. 1. down), can appear in the on/off ratio, a problem which can be addressed designing polynuclear systems and/or maximizing magnetic exchange. Eventually, a long-term goal would be to employ the tools of molecular biology to prepare metallopeptide complexes containing multiple paramagnetic ions and a certain control over their spin dynamics, since a long-term requirement for tunable memristor hardware is to be able to produce materials where one can control the response curve. Having more than one paramagnetic ion exchangecoupled to the CISS-polarized current should allow to enhance the on/off signal ratio. In turn, the response time of the memristor will be directly related to the spin relaxation time of the metal ions. Additionally, there is the possibility of influencing the overall shape of the response curve as a natural consequence of having (i) different magnetic coupling strengths between the spins of the CISS-polarized current and each of the distinct paramagnetic sites and/or (ii) different spin dynamics for the different sites. Either one of the mechanisms will mean that, for a given (externally controlled) time dependence of the external voltages, each local spin will display a different time dependence, with distinct characteristic switching times between its high-resistance state to its low-resistance state. The different combinations of high- and low-resistance states within the same molecule, which shall be controllable by the voltage history, can be expected to influence the curve shape. Importantly, to achieve these desirable goals one would not require coordination of different metals, rather, 
both the magnetic coupling with an external field and the spin dynamics of a single metal ion can be controlled up to a certain point by adjusting the coordination sphere, as has been shown in the field of single-ion magnets. 52

Having established the long-term roadmap towards the use of paramagnetic metallopeptides to achieve multistate memristive behavior, in the next sections we will discuss the state of the art and the main parts of the work that remains to be done: (a) theoretical modelling of the different physical phenomena involved and (b) experimental pathways to obtain the required systems.

\section{Challenges in theoretical modelling of CISS and spin dynamics in metallopeptides}

The theoretical effort ahead us, from the point of view of the chemical physics would be double: to determine the origin of CISS effect and its relation with the helical structure and to be able to model spin dynamics in metallopeptides. Firstly, we need to better understand the CISS effect, in particular for the case of peptides. What are the shortest sequences that will produce the maximum spin selectivity? Secondly, we need to understand spin dynamics better, and in particular how to control them in metallopeptides. How to design coordination environments that achieve the desired spin relaxation times, without compromising the magnetic exchange between the electric current and the paramagnetic center?

The CISS effect is a insufficiently explored field from the theoretical standpoint, and not even the fundamental physical processes at the root of this effect have been definitely determined yet. Currently, a few approaches have been proposed to study this effect, although they are still very preliminar. ${ }^{53}$ Although most of the theoretical effort has focused in uncovering the role of spin-orbit coupling (SOC) in the CISS effect, a quantum mechanical calculation considering SOC explicitly is still absent. As a basic preliminar approach, the group of Mujica developed a chiral electron transport theory based on assigning different spin-scattering properties to L- and D- enantiomers. ${ }^{53]}$ Other approaches such as the one proposed by Gutierrez et al., are based on the small dispersion and low mobility of the charges, which result in an unconventional Rashba-like effective spin-orbit interaction. ${ }^{57}$ At the same time, progress is being made in terms of operation principles and design guidelines for chirality-based spintronic nanodevices and technologies. 66 A better understanding and design of these chiral molecular materials will pave the way for obtaining organic spintronic devices where the neuromorphic features could be adjusted by molecular design.

While modelling the CISS effect will allow improving the coupling of the external voltage with the spin state, and thereby optimizing the on/off response in terms of the electrical resistance of the metallopeptide, the time dependence of said response requires modelling the spin dynamics, which in fact are responsible of the memristive properties. Indeed, the molecular design of the memristive behavior requires of control not just over the static spin Hamiltonian, but also over the magnetic response of metallopeptides in short times. Experimentally, there is a wide experience in employing different types of spin-tagged biomolecules in electron paramagnetic resonance, ${ }^{67}$ however this work has been focused on the dynamics of the nuclear spins, 68 and only secondary attention has been paid to the dynamics of the electron spin itself. The fields where electron spin dynamics have been explored in depth are molecular spin qubits and single-molecule magnets, 5269 so these are the fields where the relevant tools will be found. Among the limited work that has been done in time-dependent theoretical studies of magnetic metallopeptides, we were recently able to estimate time-dependent spin states between the femtosecond and the microsecond time scales through a combination of molecular dynamics and the SIMPRE code 70 (see Fig. 5). Further work will be needed for an actual prediction of the time-dependent spin dynamics as a function of the molecular design, and, in our case, of the applied voltage.
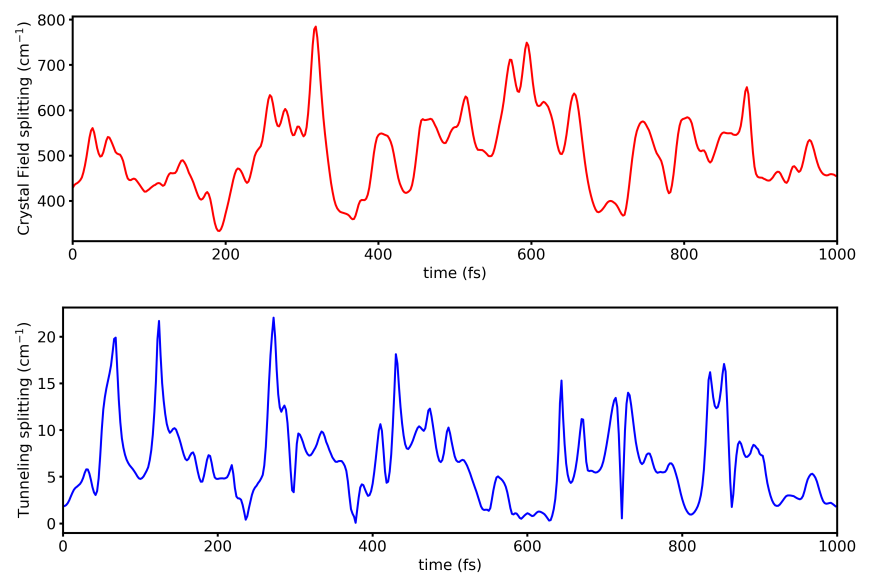

Fig. 5 Calculated time dependence of selected spin states of a TbLBT complex during a 1 ps trajectory at $300 \mathrm{~K}$ (adapted from ${ }^{70}$ ). Molecular vibrations were estimated to cause, via the crystal field, sudden changes of over $100 \%$ in the energy differences between different spin states.

Up: Energy of the upmost excited state within the $J=6$ ground manifold, i.e. total crystal field splitting. Down: energy of the first excited state i.e. tunneling splitting.

In addition, while lanthanoid complexes are amongst the most promising compounds in molecular magnetism, their organization remains an open problem, so we proposed to employ LBTs for an extremely specific and spatially-resolved organisation of lanthanoid ions as magnetic components in nanometric devices, in the context of quantum computing. Motivated by this goal, in that germinal work we employed the SIMPRE software package to evaluate our proposal theoretically for 63 different combinations of LBT peptides and lanthanide ions. 72 The calculations performed in that work allowed a preliminary estimate of the crystal field created by these polypeptides, and thus an orderof-magnitude prediction of the spin behavior in analogous complexes. In a subsequent work, we explored how different peptidic sequences can alter the spin energy levels of a complex between a LBT and a lanthanide ion:71 a limited exploration demonstrated a change in the ground state merely due to the exchange between the positions of two coordinating aminoacids, asparagine and aspartate, in the case of $\mathrm{Nd}^{3+}$ (see Fig. 6). This serves as a "proof of principle" demonstration of the possibility of effecting significant control over the spin properties of lanthanides within the 

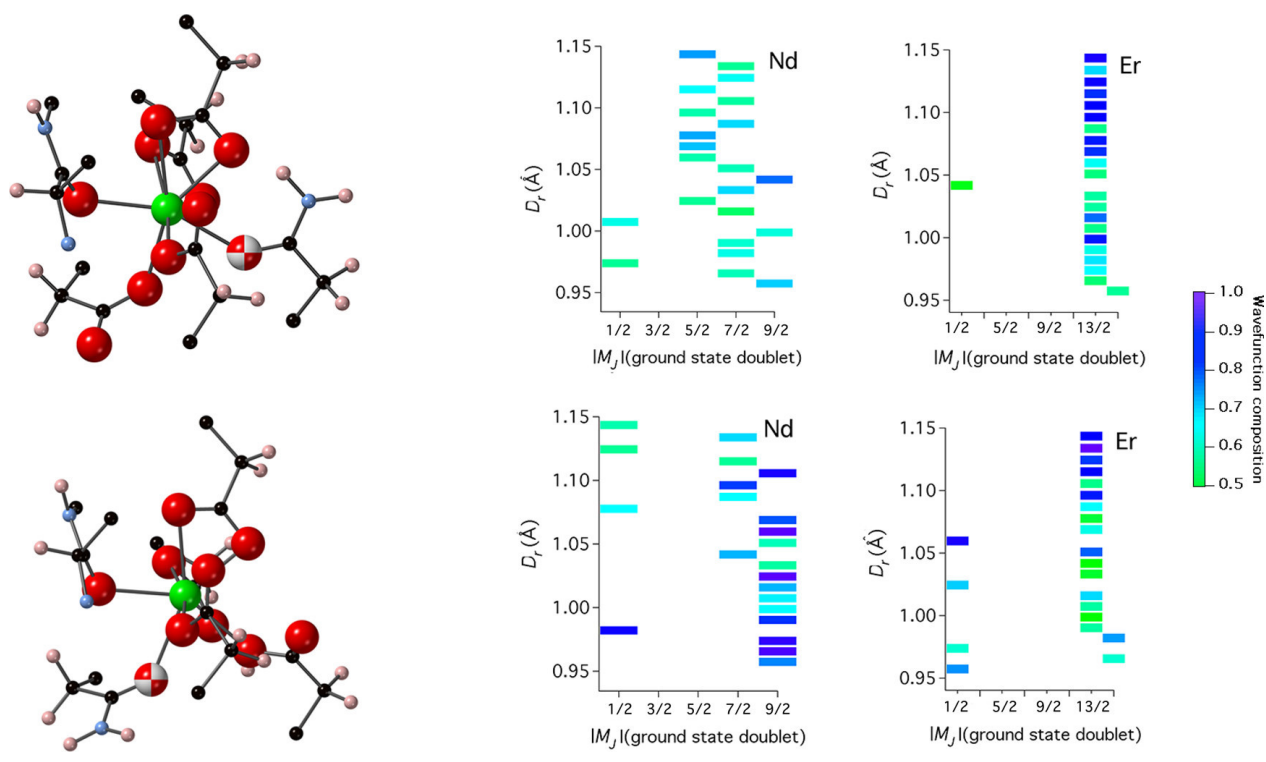

Fig. 6 Alteration of the peptidic sequence affects the charge distribution and thus the ground doublet (reused with permission from 71 ). Top and bottom rows refer to original sequence YIDTNNDGWYEGDELLA (employing the one-letter code for aminoacids) and mutant YINTDNDGWYEGDELLA, respectively. Left: coordination environment, Ln (green), O ( red), N (blue), C (black), and H (pink), highlighting the position of the carboxylate (D) vs carboxamide oxygens $(\mathrm{N})$; right: estimation of the ground doublet employing the REC model for $\mathrm{Nd}^{3+}$ and for $\mathrm{Er}^{3+}$.

metallopeptide framework by employing molecular biology tools, which is a first step towards the long-term goal of adjusting their spin relaxation times as required to achieve the desired memristive behavior.

\section{Designing metallopeptide-based tunable memris- tors}

Having established the potential of mononuclear metallopeptides acting as memristive hardware, the next step is working towards multi-spin molecular devices to enhance the on-off signal ratio. Additionally, this could serve to adjust up to a point the overall shape of the response curve, if this is required for a given application. Let us assume a multi-spin biomolecule, where the different spins present different easy axes of magnetization and different relaxation dynamics. This biomolecule bearing multiple spins could be magnetically polarized via the application of a spin current. The result would be a tunable multistate memristive component as the total resistance would depend on the intermediate spin states of the total ensemble. In that situation, it is obvious that different relative orientation of the different spins and their easy axes of magnetization would result in different memristive behavior. It is equally clear that, for any desired application, some of these spin structures will be better suited than others. What we intend to do here is merely to show how it is possible to link a rather long chain of two different kinds of lanthanide coordination environments, disregarding for the moment the key problem of their positions and orientations, which is related to a wise design of the linkers (see SI section S1).

This molecular biology method allows the extension of the synthesis for longer sequences, acommodating a high number of spins if needed. As an example and with the purpose of illustrating the synthetic feasibility of this idea, we discuss herein two ex- amples: a minimal case of a double spin with two distinct coordination environments (see SI section S2) and a more complicated asymmetric chain of 9 spins (nonanuclear metallopeptide) with a spin-spin separation of about $2 \mathrm{~nm}$ and in an arbitrarily chosen sequence of coordination environments (see SI section S3). Beyond the LBT sequence YIDTNNDGWYEGDELLA mentioned above, several peptides that also bind lanthanides have sequences that are closely related (see SI Table S1). The key here will be to combine two or more instances of LBT, with differences in the coordination environment: one will be able to combine in a single molecule two significantly different coordination environments, effectively achieving a dissimetric dinuclear lanthanide complex, ensuring an adjustable response curve to rapidly changing voltage. As an assymetric double-spin metallopeptidic prototype we employed a novel fusion of two known lanthanide binding subunits with the mentioned mutation in the coordination sphere (YIDTDNDGWYEGDELYIDTNNDGWYEGDELLA), which we note as double assymetric LBT or in short daLBT. As an example of paramagnetic polypeptides that range from mononuclear to nonanuclear in two diferent coordination environments, an arbitrary DNA sequence coding for a nonanuclear complex was designed (see DNA and protein sequence in table S3.1). Note that this allows one to design a high nuclearity system with no need to respect a simple symmetry, as it would often happen in coordination chemistry. Amplified DNA of the chosen sequence could be then sub-cloned, fused to a GST purification protein, and extracted from E. coli. Further details as well as reasonable choices for short peptidic sequences acting as linkers between the LBT sites can also be find on the SI. 
ggcagcggcgcgagcgcgggcTATATTGATACCAACAACGATGGCTGGTATGAAGGCGATGAACT CTGGCGgcgggcagcggegcgTATATTGATACCGATAACGATGGCTGGTATGAAGGCGATGAACT gcggcgggegcggcgTATATTGATACCAACAACGATGGCTGGTATGAAGGCGATGAACTGCTGGCG gcggcgggcgcggcgTATATTGATACCAACAACGATGGCTGGTATGAAGGCGATGAACTGCTGGCG ggc ggc gc gggcggcTATATTGATACCGATAACGATGGCTGGTATGAAGGCGATGAACTGgcggcg agcggcggCTATATTGATACCGATAACGATGGCTGGTATGAAGGCGATGAACTGgcgagcggcagc agcggcggcTATATTGATACCGATAACGATGGCTGGTATGAAGGCGATGAACT grcgagcggcag ATTGATACCAACAACGATGGCTGGTATGAAGGCGATGAACTGCTGGCGggcgegggegcgagcTAT ATTGATACCGATAACGATGGCTGGTATGAAGGCGATGAACTGtaatagtgaaattgg

Fig. 7 DNA sequence $\left(5^{\prime}\right.$ to $\left.3^{\prime}\right)$ corresponding to the proposed lanthanide-coordinating nonanuclear LBT protein.

\subsection{Further approaches for the biochemical design of polynuclear metallopeptides}

If the goal is to organise multiple spins in biomolecules and employ their spin dynamics to use them as multilevel memristors of any desired response curve, there are many possible ways to achieve this from the perspective of molecular biology. As the structure of DNA can be controlled up to the level of folding it like origami, ${ }^{73}$ and given that DNA also presents CISS effect, attaching paramagnetic lanthanide ions to DNA would be an obvious alternative. However, back in the 80 s the interaction of the lanthanide ions with nucleic acids was determined, including DNA and RNA, and it was found to be not selective enough for these purposes. ${ }^{74}$ Also there were studies of the direct interaction of lanthanoid ions with histone proteins, ${ }^{75}$ which again show limited specificity. However, LBTs are more specific, moreover it is only in LBTs that a spintronic effect has been experimentally demonstrated due to the interaction between the current flowing through the chiral biomolecule and the paramagnetic lanthanide ion. $\sqrt{49}$ For the ordering of LBTs at least two other approaches are conceivable (for a more detailed discussion, see SI section S4): (1) ordering on histone proteins tagged with LBTs + DNA, and (2) ordering on LBT-tagged proteins with repetitive sequences (see Fig. 8). In either case, a large three-dimensional organisation of spins inserted in a conductive, chiral scaffold capable of CISS is possible, and this opens the gates to on-demand design and preparation of multistate memristors based on biomolecules.
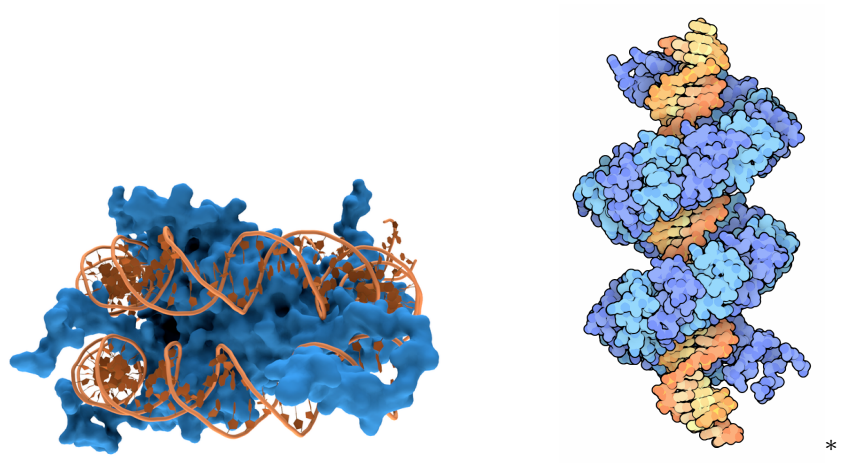

Fig. 8 Example protein structures allowing detailed organisation of peptide-coordinated lanthanoid spins in 3D space. Left: a histone octamer (nucleosome), with DNA wrapped around it. Right: TALE protein, wrapped around DNA.

\section{Conclusions}

We discussed here how the combination of molecular spintronics with Chirality Induced Spin Selectivity in designer biomolecules is a potentially fertile field for the design and preparation of memristive hardware, which in turn is a technological step towards widespread neuromorphic computing. In a nutshell, paramagnetic peptides offer a path for tuning the memristive voltagecurrent pinched hysteresis loop in terms of slope, area and response to driving voltage frequency merely by changing the peptide sequence. Crucial next steps from the theoretical side include the predictive modelling for the CISS effects in peptides as a function of their secondary structure and for the time-dependence of spin states in metallopeptides as a function of the coordination sphere. Experimentally, the first generation of memristive metallopeptides still needs to be characterized as such, but progress towards the design of the next generation is being made.

\section{Conflicts of interest}

There are no conflicts to declare.

\section{Acknowledgements}

The research reported here was supported by the European Union (ERC-CoG DECRESIM 647301 and COST-MOLSPINCA15128 Molecular Spintronics Project), the Spanish MINECO (Grant CTQ2017-89528, and Excellence Unit María de Maeztu no. CEX2019-000919-M), the Generalitat Valenciana (Prometeo Program of Excellence), and the Universitat de València (PRECOMP14-202646). S.C.-S. thank the Spanish MINECO for a Juan de la Cierva-Incorporacion grant. We kindly acknowledge Rafael Sanjuan's lab for their space, reagents and counsel.

\section{Notes and references}

$1 \mathrm{~J}$. Von Neumann, Reprinted in The Origins of Digital Computers Selected Papers, , 1975, 355-364.

2 E. Nature, Nat, 2018, 554, 145-146.

3 D. Drubach, The brain explained, Prentice Hall, 2000.

4 M. Prezioso, F. Merrikh-Bayat, B. Hoskins, G. C. Adam, K. K. Likharev and D. B. Strukov, Nature, 2015, 521, 61-64.

5 L. Chua, IEEE Transactions on circuit theory, 1971, 18, 507519.

6 G. W. Burr, R. M. Shelby, A. Sebastian, S. Kim, S. Kim, S. Sidler, K. Virwani, M. Ishii, P. Narayanan, A. Fumarola et al., Advances in Physics: X, 2017, 2, 89-124.

7 S. Pi, C. Li, H. Jiang, W. Xia, H. Xin, J. J. Yang and Q. Xia, Nature nanotechnology, 2019, 14, 35-39.

8 D. B. Strukov, G. S. Snider, D. R. Stewart and R. S. Williams, nature, 2008, 453, 80-83.

9 J. Torrejon, M. Riou, F. A. Araujo, S. Tsunegi, G. Khalsa, D. Querlioz, P. Bortolotti, V. Cros, K. Yakushiji, A. Fukushima et al., Nature, 2017, 547, 428-431.

10 A. Chanthbouala, A. Crassous, V. Garcia, K. Bouzehouane, S. Fusil, X. Moya, J. Allibe, B. Dlubak, J. Grollier, S. Xavier et al., Nature nanotechnology, 2012, 7, 101-104.

11 M. Romera, P. Talatchian, S. Tsunegi, F. A. Araujo, V. Cros, P. Bortolotti, J. Trastoy, K. Yakushiji, A. Fukushima, H. Kubota et al., Nature, 2018, 563, 230-234.

12 A. Mizrahi, T. Hirtzlin, A. Fukushima, H. Kubota, S. Yuasa, 
J. Grollier and D. Querlioz, Nature communications, 2018, 9, $1-11$.

13 C. D. Wright, P. Hosseini and J. A. V. Diosdado, Advanced Functional Materials, 2013, 23, 2248-2254.

14 A. Sebastian, T. Tuma, N. Papandreou, M. Le Gallo, L. Kull, T. Parnell and E. Eleftheriou, Nature Communications, 2017, 8, 1-10.

15 H. Al-Bustami, G. Koplovitz, D. Primc, S. Yochelis, E. Capua, D. Porath, R. Naaman and Y. Paltiel, Small, 2018, 14, 1801249.

16 S. Goswami, A. J. Matula, S. P. Rath, S. Hedström, S. Saha, M. Annamalai, D. Sengupta, A. Patra, S. Ghosh, H. Jani et al., Nature materials, 2017, 16, 1216-1224.

17 A. A. Zakhidov, B. Jung, J. D. Slinker, H. D. Abruña and G. G. Malliaras, Organic Electronics, 2010, 11, 150-153.

18 B. Mohammad, M. Abi Jaoude, V. Kumar, D. M. Al Homouz, H. A. Nahla, M. Al-Qutayri and N. Christoforou, Nanotechnology Reviews, 2016, 5, 311-329.

19 Y. Li, Z. Wang, R. Midya, Q. Xia and J. J. Yang, Journal of Physics D: Applied Physics, 2018, 51, 503002.

20 J. Roncali, P. Leriche and P. Blanchard, Advanced Materials, 2014, 26, 3821-3838.

21 C. Zhong, C. Duan, F. Huang, H. Wu and Y. Cao, Chemistry of materials, 2011, 23, 326-340.

22 S. Allard, M. Forster, B. Souharce, H. Thiem and U. Scherf, Angewandte Chemie International Edition, 2008, 47, 40704098.

23 X.-H. Zhu, J. Peng, Y. Cao and J. Roncali, Chemical Society Reviews, 2011, 40, 3509-3524.

24 Y. Park, M.-K. Kim and J.-S. Lee, Journal of Materials Chemistry C, 2020.

25 S. Cho, C. Yun, S. Tappertzhofen, A. Kursumovic, S. Lee, P. Lu, Q. Jia, M. Fan, J. Jian, H. Wang et al., Nature communications, 2016, 7, 1-10.

26 M.-J. Lee, C. B. Lee, D. Lee, S. R. Lee, M. Chang, J. H. Hur, Y.-B. Kim, C.-J. Kim, D. H. Seo, S. Seo et al., Nature materials, 2011, 10, 625-630.

27 A. Chanthbouala, V. Garcia, R. O. Cherifi, K. Bouzehouane, S. Fusil, X. Moya, S. Xavier, H. Yamada, C. Deranlot, N. D. Mathur et al., Nature materials, 2012, 11, 860-864.

28 S.-H. Yang, Applied Physics Letters, 2020, 116, 120502.

29 J. R. Brandt, F. Salerno and M. J. Fuchter, Nature Reviews Chemistry, 2017, 1, 0045.

30 O. B. Dor, S. Yochelis, S. P. Mathew, R. Naaman and Y. Paltiel, Nature communications, 2013, 4, 1-6.

31 G. Koplovitz, D. Primc, O. Ben Dor, S. Yochelis, D. Rotem, D. Porath and Y. Paltiel, Advanced Materials, 2017, 29, 1606748.

32 Y. Li, Y. Zhong, L. Xu, J. Zhang, X. Xu, H. Sun and X. Miao, Scientific reports, 2013, 3, 1619.

33 R. Naaman and L. Sanche, Chemical reviews, 2007, 107, 1553-1579.

34 I. Carmeli, V. Skakalova, R. Naaman and Z. Vager, Angewandte Chemie, 2002, 114, 787-790.
35 D. Mishra, T. Z. Markus, R. Naaman, M. Kettner, B. Göhler, H. Zacharias, N. Friedman, M. Sheves and C. Fontanesi, Proceedings of the National Academy of Sciences, 2013, 110, 14872-14876.

36 P. Roy, N. Kantor-Uriel, D. Mishra, S. Dutta, N. Friedman, M. Sheves and R. Naaman, ACS nano, 2016, 10, 4525-4531.

37 S. Ray, S. Daube, G. Leitus, Z. Vager and R. Naaman, Physical review letters, 2006, 96, 036101.

38 A. C. Aragonès, D. Aravena, J. I. Cerdá, Z. Acís-Castillo, H. Li, J. A. Real, F. Sanz, J. Hihath, E. Ruiz and I. Díez-Pérez, Nano Letters, 2016, 16, 218-226.

39 A. C. Aragonès, D. Aravena, F. J. Valverde-Muñoz, J. A. Real, F. Sanz, I. Díez-Pérez and E. Ruiz, Journal of the American Chemical Society, 2017, 139, 5768-5778.

40 P. T. Wingfield, Current protocols in protein science, 2015, 80, 6-1.

41 S. H. Sternberg and J. A. Doudna, Molecular cell, 2015, 58, 568-574.

42 R. Rogawski and A. E. McDermott, Archives of biochemistry and biophysics, 2017, 628, 102-113.

43 D. Goldfarb, Physical Chemistry Chemical Physics, 2014, 16, 9685-9699.

44 A. R. Chandrasekaran, Journal of Chemical Technology \& Biotechnology, 2016, 91, 843-846.

45 K. J. Franz, M. Nitz and B. Imperiali, ChemBioChem, 2003, 4, 265-271.

46 M. Nitz, K. J. Franz, R. L. Maglathlin and B. Imperiali, ChemBioChem, 2003, 4, 272-276.

47 K. N. Allen and B. Imperiali, Current opinion in chemical biology, 2010, 14, 247-254.

48 F. C. Mascali, H. V. Ching, R. M. Rasia, S. Un and L. C. Tabares, Angewandte Chemie International Edition, 2016, 55, 1104111043.

49 R. Torres-Cavanillas, G. Escorcia-Ariza, I. Brotons Alcázar, R. Sanchis-Gual, P. C. Mondal, L. E. Rosaleny, S. M. GiménezSantamarina, M. Sessolo, M. Galbiati, S. Tatay et al., Journal of the American Chemical Society, 2020.

50 H. Lu, J. Wang, C. Xiao, X. Pan, X. Chen, R. Brunecky, J. J. Berry, K. Zhu, M. C. Beard and Z. V. Vardeny, Science advances, 2019, 5, eaay0571.

51 U. Huizi-Rayo, J. Gutierrez, J. M. Seco, V. Mujica, I. DiezPerez, J. M. Ugalde, A. Tercjak, J. Cepeda and E. San Sebastian, Nano Letters, 2020, Article ASAP.

52 C. A. Goodwin, F. Ortu, D. Reta, N. F. Chilton and D. P. Mills, Nature, 2017, 548, 439-442.

53 S. Yeganeh, M. A. Ratner, E. Medina and V. Mujica, The Journal of chemical physics, 2009, 131, 014707.

54 P. Roushan, J. Seo, C. V. Parker, Y. S. Hor, D. Hsieh, D. Qian, A. Richardella, M. Z. Hasan, R. J. Cava and A. Yazdani, Nature, 2009, 460, 1106-1109.

55 A.-M. Guo and Q.-f. Sun, Physical review letters, 2012, 108, 218102.

56 H. S. Mandal and H.-B. Kraatz, The journal of physical chemistry letters, 2012, 3, 709-713. 
57 R. Gutierrez, E. Díaz, R. Naaman and G. Cuniberti, Physical Review B, 2012, 85, 081404.

58 E. Medina, L. A. González-Arraga, D. Finkelstein-Shapiro, B. Berche and V. Mujica, The Journal of Chemical Physics, 2015, 142, 194308.

59 S. Varela, V. Mujica and E. Medina, Physical Review B, 2016, 93, 155436.

60 A. C. Aragonès, E. Medina, M. Ferrer-Huerta, N. Gimeno, M. Teixidó, J. L. Palma, N. Tao, J. M. Ugalde, E. Giralt, I. DíezPérez et al., Small, 2017, 13, 1602519.

61 A. Kumar, E. Capua, M. K. Kesharwani, J. M. Martin, E. Sitbon, D. H. Waldeck and R. Naaman, Proceedings of the National Academy of Sciences, 2017, 114, 2474-2478.

62 V. V. Maslyuk, R. Gutierrez, A. Dianat, V. Mujica and G. Cuniberti, The journal of physical chemistry letters, 2018, 9, 54535459.

63 F. Tassinari, D. R. Jayarathna, N. Kantor-Uriel, K. L. Davis, V. Varade, C. Achim and R. Naaman, Advanced Materials, 2018, 30, 1706423.

64 S. Dalum and P. Hedegård, Nano letters, 2019, 19, 52535259.

65 R. Naaman, Y. Paltiel and D. H. Waldeck, Nature Reviews Chemistry, 2019, 3, 250-260.
66 X. Yang, C. H. van der Wal and B. J. van Wees, Nano Letters, 2020, 20, 6148-6154.

67 W. R. Lindemann and J. H. Ortony, Self-assembling Biomaterials, Elsevier, 2018, pp. 275-289.

68 A. C. Harnden, D. Parker and N. J. Rogers, Coordination Chemistry Reviews, 2019, 383, 30-42.

69 A. Lunghi and S. Sanvito, Science advances, 2019, 5, eaax7163.

70 L. E. Rosaleny, K. Zinovjev, I. Tuñón and A. Gaita-Ariño, Physical Chemistry Chemical Physics, 2019, 21, 10908-10913.

71 L. E. Rosaleny, S. Cardona-Serra, L. Escalera-Moreno, J. J. Baldoví, V. Gołebiewska, K. Wlazło, P. Casino, H. Prima-García, A. Gaita-Ariño and E. Coronado, The journal of physical chemistry letters, 2018, 9, 4522-4526.

72 L. E. Rosaleny and A. Gaita-Ariño, Inorganic Chemistry Frontiers, 2016, 3, 61-66.

73 P. W. Rothemund, Nature, 2006, 440, 297-302.

74 J. R. Morrow and C. M. Andolina, Interplay between Metal Ions and Nucleic Acids, Springer, 2012, pp. 171-199.

75 D. S. Gross, S. W. Rice and H. Simpkins, Biochimica et Biophysica Acta (BBA)-Nucleic Acids and Protein Synthesis, 1981, 656, 167-176. 